\title{
Fuzzy AHP-based Comprehensive Evaluation for Smart Grid in Energy Internet Systems
}

\author{
Ying Ma ${ }^{\mathrm{a}, \mathrm{b}}$, Yichang $\mathrm{Li}^{\mathrm{a}}$, Shunzhi Zhu ${ }^{\mathrm{a}, \mathrm{b}}$, Nan Qin ${ }^{\mathrm{a}}$, Guang Zhao ${ }^{\mathrm{c}}$, and Chao Huang ${ }^{\mathrm{c}, *}$ \\ ${ }^{a}$ Xiamen University of Technology, Xiamen, 361024, China \\ ${ }^{b}$ Key Laboratory of Data Mining and Intelligent Recommendation, Fujian Province University, Xiamen, 361024, China \\ 'Xiamen Great Power GeoInformation Technology Co., Ltd, Xiamen, 361008, China
}

\begin{abstract}
Reliability validation is an important research field in smart grid and involves the comprehensive consideration of various evaluation factors. Because it is difficult to accurately define the indicators of qualitative analysis, the fuzzy comprehensive evaluation method is introduced to evaluate each index and obtain reasonable, comprehensive and effective evaluation results. A novel model evaluation method called fuzzy analytic hierarchy process (FAHP) is proposed for smart grid in the area of energy internet, and it considers the interaction among the indices. The solution estimates the index weight through the analytic hierarchy process and builds the evaluation model using the fuzzy comprehensive evaluation method based on the hierarchy process to assess the performance of the evaluation model with fuzziness. Then, the effectiveness of the method for smart grid is verified by numerical examples. The fuzzy method proposed will provide an alternative technique for assessing different aspects of interaction in smart grid.
\end{abstract}

Keywords: reliability validation; evaluation model; fuzzy analytic hierarchy process; smart grid

(Submitted on May 2, 2018; Revised on June 16, 2018; Accepted on July 21, 2018)

(C) 2018 Totem Publisher, Inc. All rights reserved.

\section{Introduction}

Countries and regions around the world have developed new energy targets to vigorously support the development of the new energy industry. However, with the problems of climate change, environmental pollution, resource shortage and overexploitation, the energy system based on fossil energy has been unable to support the sustainable development of the economy and society. Breakthroughs continue to be made in nuclear power, wind power, solar energy and new energy as the representative of new energy technology, but strategic decisions centered on global energy production and consumption patterns should be changed for various countries.

Online information has made great progress in this field, especially due to the integration of internet and new energy technology. Energy Internet System (EIS) was first proposed by the American scholar Jeremy Rifkin [1], in smart grid based on many major breakthroughs. EIS is considered to be the future energy infrastructure platform, able to distribute energy production consumption. It can meet many challenges, such as intermittent energy regulation and access, production, storage and allocation of customized demands. EIS includes the operation and management of power systems, electrified transportation networks and traditional networks of natural gas, but other energy systems are separate. Energy internet studies usually investigate system performance and its influencing factors using different metrics [2-6], but very few focus on the model evaluation method. Recently, the combination method based on non-fuzzy theory and multiple criteria decision making (MCDM) models have attracted increasing attention within the decision-making environment. Are they appropriate for EIS evaluation?

In this paper, we exploit the fuzzy theory method to build a faster and more effective evaluation model in the area of EIS. Unlike prior works that selected the prediction model using AHP, which cannot reflect the interaction among model

\footnotetext{
* Corresponding author.

E-mail address: 13328215727@189.com
} 
indices, we propose a novel model selection algorithm called the fuzzy analytic hierarchy process (FAHP). This algorithm considers the interaction among indices. Our solution estimates the index weight with AHP and builds the evaluation model using the fuzzy comprehensive evaluation method to assess the performance of the evaluation model with fuzziness.

\section{Related Work}

The analytic hierarchy process (AHP) is a multi-criteria decision approach for organizing and analyzing complex decisions, based on structured technique and psychology discipline. It was developed by Thomas L. Saaty in the 1970s and has been extensively studied and refined since then [7]. AHP is a multiple criteria decision-making tool that has been used in almost all applications related to decision-making [8], such as store location evaluation [9], port competitiveness evaluation [10], networks risk assessment [11], and so on.

Through the analysis of the complex decision system, it can be seen that each index is not independent of each other, and it is difficult to determine the weight of the evaluation model in the structured level. It provides a comprehensive and rational framework for structuring a decision problem by representing and quantifying its elements. The AHP is conducted through the following five steps:

- Collect information about planning decisions involved, such as principles, policies and various constraints. Based on the deep understanding on the system, determine the overall goal of the system.

- Divide the system into several grade levels by building a multi-level hierarchical structure according to the target of the differences, and realize the function.

- Determine the hierarchical structure of the correlation degree between adjacent level elements. The relative weights, i.e., importance of every level of the relevant elements were given to construct the comparative judgment matrix.

- Compute the synthetic weight of the system target to determine the overall important degree of the bottom of each element in the hierarchical structure.

- Consider the corresponding decision according to the analysis and calculation results.

At present, there are many scientific and effective project evaluation methods. Multiple factors affect the external performance of EIS projects. It is difficult to quantify the relationship between the interaction and the mutual feedback, and these factors are fuzzy and uncertain. Therefore, in view of the characteristics of the external factors of EIS projects, we investigate the fuzzy analytic hierarchy process (FAHP) to determine the weight of each evaluation index.

\section{Fuzzy Analytic Hierarchy Process Evaluation for Smart Grid in the Area of Energy Internet}

The fuzzy analytic hierarchy process (FAHP) was proposed by Professor James J. Buckley to extend hierarchical analysis for the case where participants are allowed to employ fuzzy ratios in place of exact ratios [12-13]. It is a new practical decision-making method based on the analytic hierarchy process (AHP), which is especially suitable for the complex decision system for EIS. Here, the EIS evaluation process is formulated as a multiple criteria decision-making problem under uncertainty, where the imprecise decision maker's judgements are represented as fuzzy numbers. The fuzzy modification of the AHP thus obtained is implemented for finding global scores of all possible alternatives. This makes the AHP method accurate and effective through the description of the relationship between objective things.

Let the element indices of the system be represented by $e_{1}, e_{2}, \cdots, e_{n}$, then the fuzzy judgment matrix $B=\left(a_{i j}\right)_{n \times n}$ is obtained when the quantitative value $a_{i j}$ can be expressed as the important degree factor $e_{i}$ compared to factor $e_{j}$. The fuzzy judgment matrix has the following properties:

$$
a_{i j}=\left\{\begin{array}{l}
0.5, e_{i} \text { are equal important } \\
0.6, e_{i} \text { is slightly more important than } e_{j} \\
0.7, e_{i} \text { is obvious more important than } e_{j} \\
0.8, e_{i} \text { is fairly more important than } e_{j} \\
0.9, e_{i} \text { is extremely more important than } e_{j}
\end{array}\right.
$$


From Equation (1), the value $a_{i j}=0.5$ means that $a_{i}$ is as important as itself. When $a_{i j} \in[0.1,0.5], e_{i}$ is less important than $e_{j}$. When $a_{i j} \in[0.5,0.9], e_{i}$ is more important than $e_{j}$. Then, we can get the fuzzy judgment matrix:

$$
A=\left(a_{i j}\right)_{n \times n}=\left(\begin{array}{cccl}
a_{11} & a_{12} & \ldots & a_{1 n} \\
a_{21} & a_{22} & \ldots & a_{2 n} \\
\vdots & \vdots & \ddots & \vdots \\
a_{n 1} & a_{n 2} & \cdots & a_{n n}
\end{array}\right)
$$

The triangular fuzzy number has been used here:

$$
F(x)= \begin{cases}(x-L) /(m-L), & L \leq x \leq m \\ (P-x) /(P-L), & m \leq x \leq P \\ 0, & \text { others }\end{cases}
$$

Where the most possible value for the fuzzy number $F$ is $m$, and $L$ and $P$ are the left and right boundaries. Elements of this collection have no subordinate relations beyond the borders. Deriving a general formula for solving the fuzzy complementary judgment matrix, the formula contains the excellent properties of the fuzzy consistent judgement matrix. Its judgement information requires only a small amount of calculation. It is convenient for computer programming and practical application. The formula for solving the weight of the fuzzy complementary judgment matrix is as follows:

$$
W_{i}=\frac{\sum_{j=1}^{n} a_{i j}+\frac{n}{a}-1}{n(n-1)}
$$

To consider the weight and whether it is appropriate or not, we need to carry out the coincidence test. When the offset consistency is too large, it has been proven that the calculation result of the weight vector is not reliable. The principle of consistency of the fuzzy judgment matrix is derived to test the consistency principle.

Definition 1: Let $A=\left(a_{i j}\right)_{n \times n}$ and $B=\left(b_{i j}\right)_{n \times n}$ both represent the fuzzy judgement matrix. The compatibility indication for $A$ and $B$ is:

$$
I(A, B)=\frac{1}{n^{2}} \sum_{j=1}^{n} \sum_{i=1}^{n} a_{i j} b_{i j}-1
$$

Definition 2: $W=\left(W_{1}, W_{2}, \cdots, W_{n}\right)^{T}$ is the weight vector of the fuzzy judgement matrix $A$, where $\sum_{i=1}^{n} W_{i}=1, W_{i} \geq 0$, $(i=1,2, \cdots, n)$. Let $W_{i j}=W_{i} W_{j}+W_{j},(i, j=1,2, \cdots, n)$, then the Eigen matrix of the judgment matrix $A$ is:

$$
W=\left(W_{i j}\right)_{n \times n}
$$

For the decision maker $A$, the judgment matrix satisfies the consistency condition when the consistency index $I(A, W) \leq A$. The smaller the $A$, the higher the consistency of the fuzzy judgment matrix required by the decision maker. For real applications, some experts give the comparison judgment matrix on factor set $X$, with $k=1,2, \cdots, m$.

$$
A_{k}=\left(a_{i j}^{(k)}\right)_{n \times n}
$$


Where $k=1,2, \cdots, m$. From these fuzzy complementary judgment matrixes, we can obtain the weighting set $W^{(k)}=\left(w_{1}^{(k)}, w_{1}^{(k)}, \cdots, w_{n}^{(k)}\right),(k=1,2, \cdots, m)$. The consistency test of the fuzzy complementary judgment matrix is completed using the following two steps:

(1) The consistency of M judgment matrix $A_{k}$ :

$$
I\left(A_{k}, W^{(k)}\right) \leq A, \quad k=1,2, \cdots, m
$$

(2) The satisfactory consistency between judgment matrices:

$$
I\left(A_{i}, A_{j}\right) \leq A, \quad i \neq j, \quad i, j=1,2, \cdots, m
$$

Where $1 \leq i \leq m, 1 \leq j \leq p, m$ is the number of the models, and $p$ is the number of the performance metrics. It can be proven that if the consistency of the fuzzy complementary judgment matrices $A_{k}(k=1,2, \cdots, m)$ are acceptable, then their corresponding judgment matrices are also acceptable. The weight vector is:

$$
W^{(k)}=\left(W_{1}, W_{2}, \cdots, W_{n}\right)
$$

Where $W_{i}=\frac{1}{n} \sum_{k=1}^{n} W_{i}^{(k)},(i=1,2, \cdots, n)$. As long as both Equation (1) and (2) meet, the means of the $m$ weight set can be accepted as reasonable and reliable weights for the indices.

According to the weight, the result is tested by consistency, as in the following formula:

$$
\begin{gathered}
B W=\lambda W \\
C I=\frac{\lambda_{\max }-n}{n-1}
\end{gathered}
$$

In the formula, $B$ is the judgment matrix of each order, and the percent is the corresponding element in the judgment matrix. $A$ is the characteristic root of the judgment matrix. If $C I<0.10$, then we use the consistency check. The weights of each evaluation index are calculated respectively. Finally, the weight of each evaluation index and the normalized score $W_{i}$ are given. The quantitative indicators $x_{i}$ in the index system of standardized treatment can regulate the $y_{i}$ value. The standard value $i$ of the index $c_{i}$ is obtained through the cost minimized means, as follows:

$$
\begin{gathered}
y_{i}=\frac{x_{i}-\min _{1 \leq j \leq n} x_{j}}{\max _{1 \leq j \leq n} x_{j}-\min _{1 \leq j \leq n} x_{j}} \\
a_{i j}= \begin{cases}y_{i}, & \max e_{i} \\
1-y_{i}, & \min e_{i}\end{cases}
\end{gathered}
$$

In order to conduct a comprehensive score, the comprehensive score of $G$ should be obtained to determine whether the project should make appropriate adjustments to achieve the minimum. The comprehensive score $U$ can be computed as follows:

$$
G=\sum_{i=1}^{n} \tau_{i} U_{i}
$$




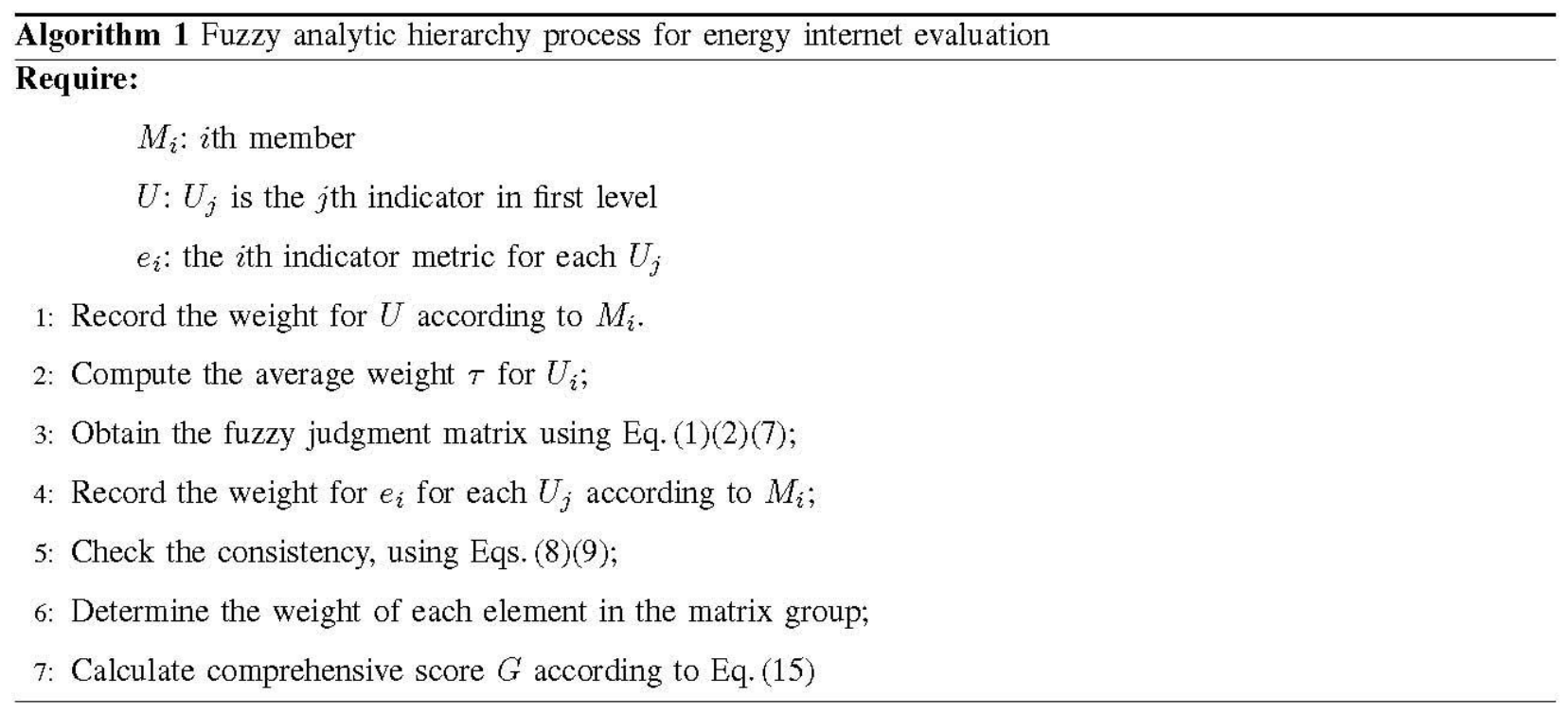

Figure 1. Algorithm for fuzzy analytic hierarchy process for energy internet evaluation

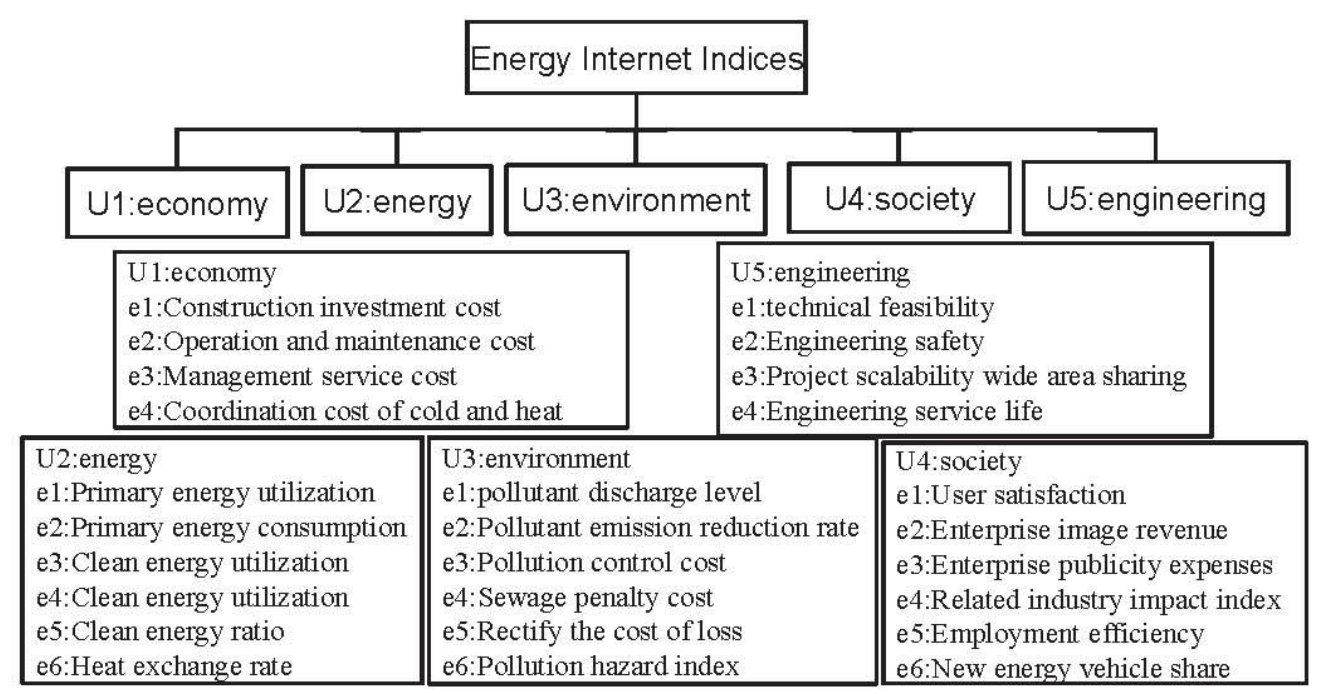

Figure 2. The first and second levels of energy internet indices

\section{Empirical Research}

Taking smart grid in the area of energy internet as an example, experts from energy engineering teams provide relative weights using the AHP method. To make the weight more reliable and obtain a stable final judgment matrix and weight coefficient, the consistency of the matrix is checked. We calculate the average score of each member in the data processing. After the data processing, the weight coefficient is obtained:

$$
\tau=\left[\begin{array}{l}
0.15 \\
0.24 \\
0.16 \\
0.33 \\
0.12
\end{array}\right]
$$


$\tau$ is the weight coefficient of the first level indicators, while $A_{1}, A_{2}, A_{3}, A_{4}, A_{5}$ are the weight coefficients of the second level indicators.

$$
\begin{aligned}
& A_{1}=(0: 050 ; 0: 428 ; 0: 201 ; 0: 321) \\
& A_{2}=(0: 333 ; 0: 151 ; 0: 125 ; 0: 184 ; 0: 143 ; 0: 064) \\
& A_{3}=(0: 094 ; 0: 021 ; 0: 245 ; 0: 195 ; 0: 237 ; 0: 208) \\
& A_{4}=(0: 115 ; 0: 037 ; 0: 323 ; 0: 252 ; 0: 176 ; 0: 097) \\
& A_{5}=(0: 298 ; 0: 368 ; 0: 056 ; 0: 278)
\end{aligned}
$$

We distributed 500 questionnaires about the Chinese southern power grid of energy internet and received 386 valid questionnaires, with an effective rate of $95.33 \%$. The second level has 26 indicators. The relative membership degree of each is obtained from the questionnaires.

$$
\begin{aligned}
& B_{1}=\left[\begin{array}{lllll}
0.20 & 0.41 & 0.34 & 0.05 & 0 \\
0.25 & 0.50 & 0.04 & 0 & 0 \\
0.10 & 0.37 & 0.47 & 0.04 & 0 \\
0.34 & 0.31 & 0.14 & 0.22 & 0
\end{array}\right], B_{2}=\left[\begin{array}{lllll}
0.1 & 0.64 & 0.2 & 0.06 & 0 \\
0.35 & 0.56 & 0.09 & 0 & 0 \\
0.31 & 0.24 & 0.10 & 0.35 & 0 \\
0.16 & 0.20 & 0.45 & 0.20 & 0 \\
0.20 & 0.22 & 0.15 & 0.44 & 0 \\
0.45 & 0.21 & 0.20 & 0.15 & 0
\end{array}\right] \\
& B_{3}=\left[\begin{array}{lllll}
0.30 & 0.50 & 0.00 & 0.00 & 0 \\
0.40 & 0.60 & 0.00 & 0.00 & 0 \\
0.40 & 0.21 & 0.15 & 0.24 & 0 \\
0.30 & 0.23 & 0.25 & 0.21 & 0 \\
0.44 & 0.21 & 0.15 & 0.21 & 0 \\
0.15 & 0.60 & 0.20 & 0.03 & 0
\end{array}\right], \quad B_{4}=\left[\begin{array}{ccccc}
0.30 & 0.70 & 0.00 & 0.00 & 0 \\
0.30 & 0.40 & 0.10 & 0.10 & 0.10 \\
0.51 & 0.12 & 0.20 & 0.14 & 0.03 \\
0.41 & 0.24 & 0.35 & 0.00 & 0 \\
0.34 & 0.31 & 0.35 & 0.00 & 0 \\
0.42 & 0.23 & 0.14 & 0.21 & 0
\end{array}\right] \\
& B_{5}=\left[\begin{array}{lllll}
0.50 & 0.10 & 0.20 & 0.10 & 0.10 \\
0.25 & 0.35 & 0.25 & 0.11 & 0.04 \\
0.41 & 0.36 & 0.15 & 0.04 & 0.03 \\
0.22 & 0.26 & 0.15 & 0.32 & 0.05
\end{array}\right], \quad V=\left[\begin{array}{l}
95 \\
85 \\
75 \\
65 \\
50
\end{array}\right]
\end{aligned}
$$

Then, we can obtain Table 1 , by computing $U_{i}=A_{i} \times B_{i} \times V,(i=1,2,3,4,5)$. According to the weight process, the final evaluation value is:

$$
U=\tau \times\left[\begin{array}{l}
U_{1} \\
U_{2} \\
U_{3} \\
U_{4} \\
U_{5}
\end{array}\right]=81.92
$$

Table 1. The score of each index

\begin{tabular}{|c|c|c|c|c|}
\hline U1:economy & U2:energy & U3:environment & U4:society & U5:engineering \\
\hline 76.40 & 81.89 & 81.63 & 84.97 & 80.85 \\
\hline
\end{tabular}


It is concluded that since there are too few multiple criteria decision making methods for energy engineers, the interaction between the model indexes from different performance metrics may help. We are optimistic that the fuzzy theory based method can guide optimal evaluation strategies, possibly achieving accurate stability evaluation for energy internet.

\section{Conclusions}

We have proposed a new model evaluation method, FAHP, for the energy internet system. The solution first estimates the index weight using AHP and then builds the evaluation model using the fuzzy comprehensive evaluation method. This method is based on the analytic hierarchy process to assess the performance of the evaluation model with fuzziness. In the future, we will try to investigate other weight algorithms for model evaluation on additional energy internet systems.

\section{Acknowledgements}

This work was supported in part by the National Natural Science Foundation of China (Grant No. 61502404, 61672442), Natural Science Foundation of Fujian Province of China (Grant No. 2016J01326, 2016Y0079), Distinguished Young Scholars Foundation of Fujian Educational Committee (Grant No. DYS201707), International S\&T Cooperation Program (Grant No. E201402000), and Open Fund of Key Laboratory of Data Mining and Intelligent Recommendation, Fujian Province University. We thank the anonymous reviewers for their greatly helpful comments.

\section{References}

1. J. Rifkin, "The Third Industrial Revolution: How Lateral Power is Transforming Energy, the Economy, and the World," Palgrave Macmillan, New York City, 2011

2. J. Teh and I. Cotton, "Reliability Impact of Dynamic Thermal Rating System in Wind Power Integrated Network," IEEE Transactions on Reliability, Vol. 65, No. 2, pp. 1081-1089, November 2016

3. A. Arab, E. Tekin, A. Khodaei, S. K. Khator, and H. Zhu, "System Hardening and Condition-Based Maintenance for Electric Power Infrastructure Under Hurricane Effects," IEEE Transactions on Reliability, Vol. 65, No. 3, pp. 1457-1470, November 2016

4. L. Jiang, Y. Yuan, Z. Wang, and S. Wang, "Evaluation Index System and Comprehensive Evaluation Method of Energy Internet in Innovative Demonstration Area of Smart Grid," Automation of Electric Power Systems, Vol. 1, No. 28, pp. 39-45, January 2016

5. R. Teng, H. B. Li, and R. Miura, "Performance Evaluation of the Decreasing Energy and Connectivity in the Internet-Connected Mesh Networks with Biased User Mobility," IEICE Technical Report Mona Mobile Network \& Applications, 114, 2015

6. J. F. G. Fernndez, A. C. Mrquez, and M. A. Lpez-Campos, "Customer-oriented Risk Assessment in Network Utilities," Reliability Engineering \& System Safety, Vol. 147, pp. 72-83, March 2018

7. T. L. Saaty, "Optimization in Integers and Related Extremal Problems," McGraw-Hill Book Co. New York-London-Sydney, 1970

8. O. S. Vaidya and S. Kumar, “Analytic Hierarchy Process: An Overview of Applications,” European Journal of Operational Research, Vol. 169, No. 1, pp. 1-29, February 2006

9. E. KoQ and H. A. Burhan, "An Application of Analytic Hierarchy Process (AHP) in a Real World Problem of Store Location Selection," Advances in Management \& Applied Economics, Vol. 5, No. 1, pp. 41-50, January 2015

10. G. K. V. Dyck and H. M. Ismael, "Multi-Criteria Evaluation of Port Competitiveness in West Africa Using Analytic Hierarchy Process (AHP)," American Journal of Industrial \& Business Management, Vol. 5, No. 6, pp. 432-446, June 2015

11. H. Y. Tsai and Y. L. Huang, "An Analytic Hierarchy Process-based Risk Assessment Method for Wireless Networks," IEEE Transactions on Reliability, Vol. 60, No. 4, pp. 801-816, January 2011

12. J. J. Buckley, "Fuzzy Hierarchical Analysis," Fuzzy Sets \& Systems, Vol. 17, No. 3, pp. 233-247, December 1985

13. T. N. An, D. N. Long, L. H. Long, and C. N. Dang, "Quantifying the Complexity of Transportation Projects using the Fuzzy Analytic Hierarchy Process," International Journal of Project Management, Vol. 33, No. 6, pp. 1364-1376, February 2015 